\title{
Hypertriglyceridemia: An Open Door for Cancer Treatment?
}

\author{
Le Yu Khine ${ }^{\mathrm{a}, \mathrm{b}}$, Jamila A. Benmoussa ${ }^{\mathrm{a}}$, Andrew T. Thorp Jrª, Neena Chandrasekaran ${ }^{\mathrm{a}}$, \\ Thomas Forlenza ${ }^{a}$, Dennis Bloomfield ${ }^{a}$
}

Does lipid management have a significant role in cancer treatment? Could hypertriglyceridemia be an accelerating factor in certain tumors? These questions have grown from the long-standing recognition of the relationship of lipids to certain cancers. Cancers express high levels of lipid synthase, the enzyme responsible for the production of fatty acids. Lymphoma and other cancers have been found to have the ability to generate the lipids via de novo lipogenesis at a rate faster than other lipogenetic tissues [1]. Continuous de novo lipogenesis provides cancer cells with membrane building blocks, signaling lipid molecules, posttranslational modifications of proteins as well as energy supply to support rapid cell proliferation [2]. Protection of cancer cells by apoptosis by overexpression of fatty acid synthase (FASN) also helps the progression of the cancer [2].

Recent studies have documented the changes in lipid levels in the etiology and most importantly prognosis of cancer [3, 4]. There are many studies that have shown that the degree of lipid abnormality was directly related to the underlying tumor burden in addition to the abnormality in triglyceride clearance [5]. In one study, 25 patients with acute leukemia and nonHodgkin's lymphoma (NHL) were studied; all patients demonstrated an abnormality in at least one plasma lipid fraction that consisted of extremely low levels of high density lipoprotein (HDL), elevated triglycerides (TG) and elevated very low density lipoprotein (VLDL). The levels were found to be normalized after recovery [5]. Another study was performed to investigate serum lipid alterations in patients with acute lymphoblastic leukemia (ALL) at diagnosis and during remission of the disease. Plasma lipids and lipoproteins were measured at diagnosis, prior to the administration of induction treatment, and every 2 months for the first 12 months of the maintenance phase of chemotherapy in 64 patients with ALL. Nearly all patients demonstrated serum lipid alterations which included elevated TG, LDL and cholesterol and values were returned

Manuscript accepted for publication March 24, 2016

a Department of Internal Medicine, Richmond University Medical Center, Staten Island, NY, USA

bCorresponding Author: Le Yu Khine, Department of Internal Medicine, Richmond University Medical Center, Staten Island, NY, USA.

Email: leyukhinebaybay@gmail.com

doi: http://dx.doi.org/10.14740/jem342w back to normal during remission [6]. This points out the direct link between cancer progression and lipid levels. Metastasis is another interesting area in which the lipid level can correlate with disease burden. In one study, 205 Iranian patients from Semnan Oncology Clinic were studied and there was a significant association between higher levels of serum LDL and metastasis [7]. Aggressiveness of the tumor including metastasis can be highlighted by the levels of lipids. This brings up to the point of our case. We present a patient with stage IV diffuse large B-cell NHL with diffuse involvement of the bone and liver whose course was complicated with an incidental finding of hypertriglyceridemia.

A 67-year-old Caucasian male with a past medical history of chronic obstructive pulmonary disease, diabetes mellitus, hypertension, coronary artery disease, and pulmonary embolism was admitted to the hospital for severe lower back pain and significant weight loss for 1 month. He denied any familial or personal history of hypertriglyceridemia. Physical examination was remarkable for pale conjunctivae, positive straight leg test on the left lower extremity, and a limited range of motion in his right upper extremity. There was no point tenderness noted in his back but there was mild tenderness to palpation of the right chest wall. CT abdomen imaging revealed several masses in the liver which involved the lateral segment of the left lobe, the medial segment, and an illdefined mass in the right lobe posteriorly. Biopsy of the liver demonstrated evidence for diffuse large B-cell NHL and bone marrow involvement was also seen in bone marrow biopsy. Lipid profile drawn 2 years ago were found to be TG $195 \mathrm{mg} /$ dL, cholesterol 146 mg/dL, LDL 74 mg/dL, VLDL 39 mg/dL and HDL $33 \mathrm{mg} / \mathrm{dL}$. The lipid panel had changed dramatically during this admission demonstrating TG 1,154 mg/dL, cholesterol $204 \mathrm{mg} / \mathrm{dL}$, and HDL $4 \mathrm{mg} / \mathrm{dL}$, and LDL and VLDL were unable to be calculated. This unusual level of TG highlighted the aggressiveness of the tumor and more importantly the prognosis of the cancer. The patient was treated with gemfibrozil and insulin. TG levels were markedly improved from 1,154 to $690 \mathrm{mg} / \mathrm{dL}$. The patient deteriorated before initiation of chemotherapy and expired.

The issue of interest in this case is not the specific attributes of the cancer discussed but of the rise and fall of TG associated with it. The possible implications of this event may correlate with the aggressiveness of the tumor or the type of cancer and presents an intriguing possibility of manipulating this process as a means of treatment. The current standards for 
cancer treatment may possibly be improved with lipid screening tests and potential targeted adjuvant therapies targeting processes in this pathway. Lipid metabolism has long been known to be a component of the pathogenesis and aggressive potential of a given cancer.

The interesting link between lipids and cancer can possibly lead to the creation of new targeted therapies of cancer treatment era. The effect of blocking individual components of the pathways involved in the biosynthesis, uptake, or remodeling of lipids needs to be evaluated not only in the context of cancer cell proliferation and survival but also within the more complex setting of cancer cell migration, invasion, tumor angiogenesis and metastasis formation. A number of chemical inhibitors of lipid biosynthesis, most prominently inhibitors of FASN, have been investigated in preclinical cancer models or are entering clinical trials. Cerulenin and C75, both early small-molecule FASN inhibitors, have demonstrated significant antitumor activity [8]. Some of these drugs have originally been developed to treat metabolic diseases, such as diabetes mellitus, hyperlipidemia or the metabolic syndrome, and it is likely that more overlap between these disease settings and cancer will be discovered in the future. In this context, the contribution of dietary factors, such as saturated and unsaturated fatty acid, also needs to be considered - future cancer therapeutic strategies that could incorporate strict dietary regimes. Despite the substantial challenges that need to be overcome for successful drug development, a plethora of novel therapeutic opportunities are indicated [9].

Given our current knowledge of these processes we feel that further studies may demonstrate a possible correlation between lipid levels, types of lipids, and may be a quantifiable marker of tumor malignant potential and could possibly lead to earlier, more focused treatments with a potential mortality benefit. Recognition and aggressive control of lipid levels at early stages can be an open door to cancer treatment. A new era of therapeutic opportunities which can benefit our cancer patients is awaiting.

\section{Conflicts of Interest}

None.

\section{References}

1. Medes G, Thomas A, Weinhouse S. Metabolism of neoplastic tissue. IV. A study of lipid synthesis in neoplastic tissue slices in vitro. Cancer Res. 1953;13(1):27-29.

2. Zhang F, Du G. Dysregulated lipid metabolism in cancer. World J Biol Chem. 2012;3(8):167-174.

3. Cvetkovic Z, Cvetkovic B, Petrovic M, Ranic M, Debeljak-Martarcic J, Vucic V, Glibetic M. Lipid profile as a prognostic factor in cancer patients. J BUON. 2009;14(3):501-506.

4. Muntoni S, Atzori L, Mereu R, Satta G, Macis MD, Congia M, Tedde A, et al. Serum lipoproteins and cancer. Nutr Metab Cardiovasc Dis. 2009;19(3):218-225.

5. Spiegel RJ, Schaefer EJ, Magrath IT, Edwards BK. Plasma lipid alterations in leukemia and lymphoma. Am J Med. 1982;72(5):775-782.

6. Moschovi M, Trimis G, Apostolakou F, Papassotiriou I, Tzortzatou-Stathopoulou F. Serum lipid alterations in acute lymphoblastic leukemia of childhood. J Pediatr Hematol Oncol. 2004;26(5):289-293.

7. Ghahremanfard F, Mirmohammadkhani M, Shahnazari B, Gholami G, Mehdizadeh J. The Valuable Role of Measuring Serum Lipid Profile in Cancer Progression. Oman Med J. 2015;30(5):353-357.

8. Flavin R, Peluso S, Nguyen PL, Loda M. Fatty acid synthase as a potential therapeutic target in cancer. Future Oncol. 2010;6(4):551-562.

9. Baenke F, Peck B, Miess H, Schulze A. Hooked on fat: the role of lipid synthesis in cancer metabolism and tumour development. Dis Model Mech. 2013;6(6):13531363. 